\title{
Genome-wide analysis of LTR-retrotransposons in oil palm
}

Thierry Beulél ${ }^{*}$ (D, Mawussé DT Agbessi ${ }^{1}$, Stephane Dussert ${ }^{2}$, Estelle Jaligot ${ }^{1}$ and Romain Guyot ${ }^{3}$

\begin{abstract}
Background: The oil palm (Elaeis guineensis Jacq.) is a major cultivated crop and the world's largest source of edible vegetable oil. The genus Elaeis comprises two species $E$. guineensis, the commercial African oil palm and $E$. oleifera, which is used in oil palm genetic breeding. The recent publication of both the African oil palm genome assembly and the first draft sequence of its Latin American relative now allows us to tackle the challenge of understanding the genome composition, structure and evolution of these palm genomes through the annotation of their repeated sequences.

Methods: In this study, we identified, annotated and compared Transposable Elements (TE) from the African and Latin American oil palms. In a first step, Transposable Element databases were built through de novo detection in both genome sequences then the TE content of both genomes was estimated. Then putative full-length retrotransposons with Long Terminal Repeats (LTRs) were further identified in the E. guineensis genome for characterization of their structural diversity, copy number and chromosomal distribution. Finally, their relative expression in several tissues was determined through in silico analysis of publicly available transcriptome data.

Results: Our results reveal a congruence in the transpositional history of LTR retrotransposons between E. oleifera and E. guineensis, especially the Sto-4 family. Also, we have identified and described 583 full-length LTR-retrotransposons in the Elaeis guineensis genome. Our work shows that these elements are most likely no longer mobile and that no recent insertion event has occurred. Moreover, the analysis of chromosomal distribution suggests a preferential insertion of Copia elements in gene-rich regions, whereas Gypsy elements appear to be evenly distributed throughout the genome.

Conclusions: Considering the high proportion of LTR retrotransposon in the oil palm genome, our work will contribute to a greater understanding of their impact on genome organization and evolution. Moreover, the knowledge gained from this study constitutes a valuable resource for both the improvement of genome annotation and the investigation of the evolutionary history of palms.
\end{abstract}

Keywords: Oil palm, Transposable elements, Retrotransposons, LTR

\section{Background}

Transposable Elements (TEs) are mobile and parasitic nucleic acids that can be distinguished according to their respective mode of transposition. Class I elements or retrotransposons use a RNA intermediate through a "copy and paste" mechanism that may result in an exponential increase of copy number within the genome, whereas Class II elements or transposons are excised from their original genomic insertion ("cut and paste" mechanism) and transpose as DNA molecules [1]. TEs make up a significant fraction of many eukaryotic genomes and, in plants, the increase in TE content is

\footnotetext{
* Correspondence: thierry.beule@cirad.fr

'CIRAD, UMR DIADE (IRD, UM), 34394 Montpellier, France

Full list of author information is available at the end of the article
}

strongly correlated with the increase in genome sizes observed amongst Angiosperms, from $10 \%$ in Arabidopsis [2] to up to $80-85 \%$ in cereals (maize: [3]; wheat: [4]; barley: [5]; see also [6-8] for reviews).

Because of their replication mode, retrotransposons constitute the most abundant TE class. Among them, those with Long Terminal Repeats (LTRs), belonging to Gypsy and Copia super-families are largely predominant in the genomes of flowering plants $[1,9]$. When annotating TE sequences in genomes, further distinction is between autonomous and non-autonomous elements based on the presence or the absence, respectively, of both the POL and GAG coding domains that are required for transposition, regardless of whether these sequences are actually functional $[1,10]$. 
LTR-retrotransposons have significant effects on genome instability through homologous recombination between copies, with potentially long-term consequences on genome evolution $[11,12]$. LTR-retrotransposons tend to accumulate within pericentromeric heterochromatin [13] and they also contribute to the formation of functional centromeres in plants [14].

In addition to their effects on gene and genome structure, the insertion of LTR retrotransposons may also affect the regulation of nearby genes and this can in turn result in the emergence of phenotypic variation [15]. Indeed, TEs are targeted by powerful epigenetic repressive mechanisms that ensure their maintenance in a stably silenced statgenome sequences. Phylogenetic trees were based one and these TE-silencing processes share a number of components with pathways that are responsible for the epigenetic regulation of host genes expression [16]. Once an element has been transcriptionally inactivated, it accumulates mutations over time and loses the ability to transpose autonomously. However, even elements that are both transcriptionally silent and immobile can be co-opted by the host genome to provide new beneficial features for both gene regulation and genome evolution through the rewiring of regulatory networks, a phenomenon known as exaptation or molecular domestication [10, 17-20].

The epigenetic repression of TE activity can be transiently alleviated as a result of environmental stresses (heat, cold, UV light, pathogen attack...) and this reactivation, which can affect a variable fraction of the TE populations, is thought to contribute to the short-term response to changing environmental conditions [12, 15, 21-23]. Tissue culture processes, in particular, are well-known triggers of LTR-retrotransposons remobilization [24], as illustrated by the examples of Tos17 in rice [25], Tht1 [26] and Tto1 [27] in tobacco and BARE-1 in barley [28]. Ultimately, these reactivated elements can contribute to the phenomenon of somaclonal variation and promote the emergence of altered phenotypes [29-31].

The oil palm (Elaeis guineensis Jacq.) is a major cultivated crop and the world's largest source of edible vegetable oil. The genus Elaeis comprises two species: Elaeis guineensis Jacq. (Eg) originates from West Africa and Elaeis oleifera Cortés (Eo) is found in Central and South America. The inter-fertility between both species allows the use of interspecific hybrid populations in breeding programs.

The African oil palm (Elaeis guineensis) is an example of an economically important crop that is commercially propagated through the in vitro cloning of high oil-producing individuals. The unpredictable incidence of the mantled floral variant among the clonal progeny and its negative impact on oil yields [32] has prompted the search for the molecular mechanisms underlying this phenotype. The reversibility and heterogeneity of the variation have led to the hypothesis of an epigenetic origin, which has been supported by the characterization of a significant DNA hypomethylation of the mantled genome [33-35]. Because of the abovementioned well-documented relationship between TE activity and certain somaclonal variations, several attempts have been made to identify some of the LTR retrotransposons of oil palm [36,37] or to find evidence of their mobilization as a result of the genome-wide hypomethylation found in mantled tissues [38]. These studies were however inconclusive at the time, presumably due in part to the lack of genome-wide sequence information allowing only the investigation of individual elements. Therefore a thorough study of LTR retrotransposon populations throughout the oil palm genome and in connection with the mantled variation is still warranted. In order to achieve this, it is necessary to first identify and classify TEs from the recently published African oil palm genome assembly [39], as well as from the draft sequence of its Latin American relative (E. oleifera) for comparison purposes. To further facilitate data mining, software tools allowing the identification of the structural features of TEs from highthroughput sequencing data have been developed [40-42].

In the present study, we have conducted a genome-wide annotation of Transposable Elements from the publicly available African and American oil palm genome sequences, with a focus on LTR retrotransposons. As a preliminary step, we have built a Transposable Element database and analyzed the TE content for each of the two oil palm genome sequences in order to compare their respective TE populations. We have further identified putative full-length LTR retrotransposons in the E. guineensis genome and characterized their structural diversity, chromosomal distribution and estimated their evolution through time. In addition, we have analyzed their transcriptional activity in a variety of organs. Our results provide insights on the LTR retrotransposon landscape and evolution in both the oil palm genomes and constitute a valuable resource for the improvement of their respective genome assembly. Ultimately, the resulting oil palm retrotransposons dataset paves the way for further investigating the role of these elements in the mantled somaclonal variation.

\section{Results}

\section{De novo construction and analysis of consensus TE databases}

A total of 991 E. oleifera (Eo) and 846 E. guineensis (Eg) scaffolds were used for self-comparison in order to detect repeated sequences within each dataset. After clustering, 4025 and 10,193 consensus repeated sequences were selected and classified as transposable elements according to Repbase [43] for E. oleifera and E. guineensis, respectively. The complete databases are provided in Additional file 1 and a summary of their contents is presented in Additional file 2. The consensus sequences that were classified as either chimeric or potential host genes (272 and 
278 sequences, respectively) were subsequently removed from TEdenovo's output. Among the remaining 3475 Eo consensus sequences, $72 \%$ are assigned to Class I and 19 \% to Class II, whereas for Eg (9915 consensus sequences) the respective proportions are 39 and $8 \%$, respectively (Fig. 1). Remarkably, the main difference between the two sets of consensus sequences is the considerably larger proportion of unclassified repeats (NoCat) in E. guineensis (54, vs. 9 \% for E. oleifera).

Among successfully classified repeats, the most represented groups in both genomes are, in decreasing order: RXX (unclassified retrotransposons) and potentially non-autonomous retrotransposons such as LARDs and TRIMs; 58 \% for Eo; 60 \% for Eg), DTX (transposons; 20 and $16 \%$, respectively) and RLX (LTR retrotransposons; 15 and $16 \%$, respectively) (Fig. 1).

The RLX consensus sequences were further classified into lineages and families [44]. Significant sequence similarities were detected for 337 and 491 RLX consensus sequences from Eo and Eg respectively. The analysis of the resulting Neighbor-Joining trees (Fig. 2) shows that most of the LTR retrotransposon lineages that have previously been identified in other Angiosperm genomes are represented in both E. oleifera (Eo) and E. guineensis (Eg). Interestingly, lineage diversity appears to be similar between both oil palm genomes. It is also worth noting that all the LTR retrotransposon consensus sequences (RLX) identified by TEdenovo in both Elaeis genomes were classified as incomplete elements.

\section{TE abundance in oil palm genomes ( $E$. oleifera and $E$. guineensis)}

The impact of TE populations on the genome sizes of E. oleifera (Eo) and E. guineensis (Eg) was estimated [45]. The TEdenovo output for Eo masks $41.39 \%$ $(580,386,071 \mathrm{bp})$ of available genomic sequences and $55.9 \%$ when excluding unassigned nucleotides $(\mathrm{N})$ from the analysis. Similar proportions are obtained
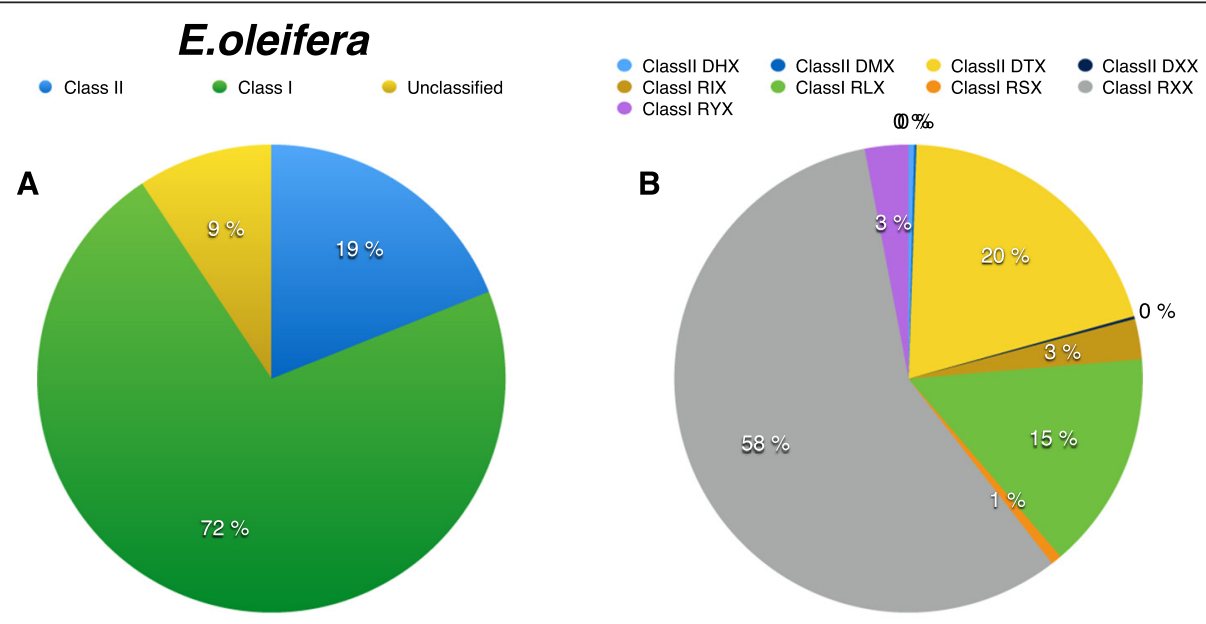

\section{E.guineensis}
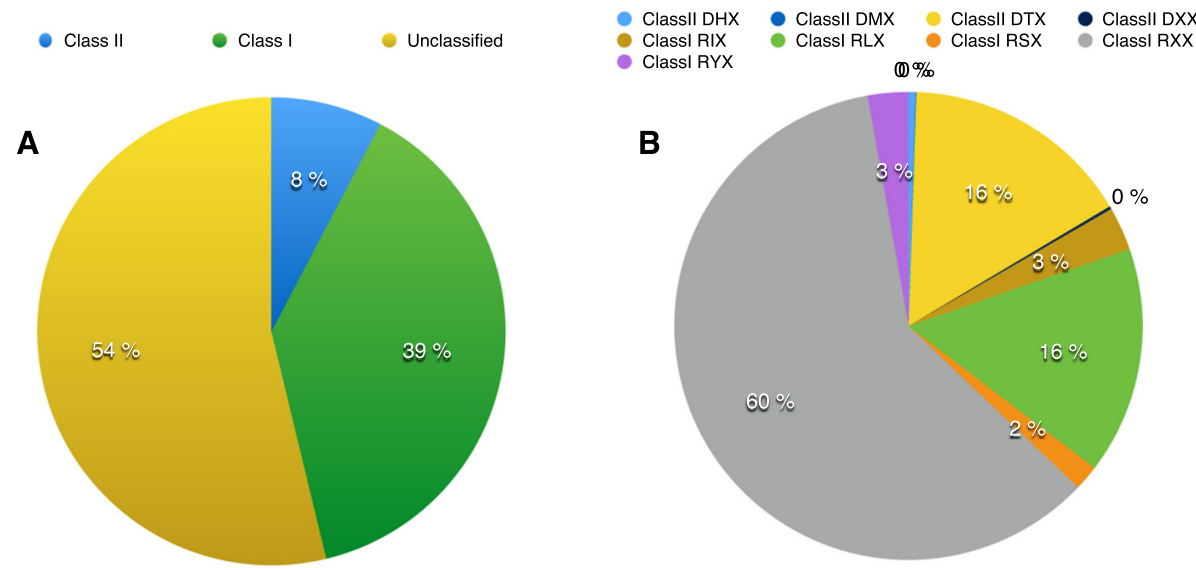

Fig. 1 Overview of the TE contents of the E. oleifera and E. guineensis genome sequences, as identified and classified by the REPET software Top panel E. oleifera; bottom panel E. guineensis. a: classes; b: orders. Class I elements: RIX LINE; RLX LTR Retrotransposons; RSX SINE; RXX unclassified and Non-autonomous Retrotransposons; RYX DIRS. Class II elements: DHX Helitron; DMX Maverick; DTX TIR Transposons; DXX MITE 


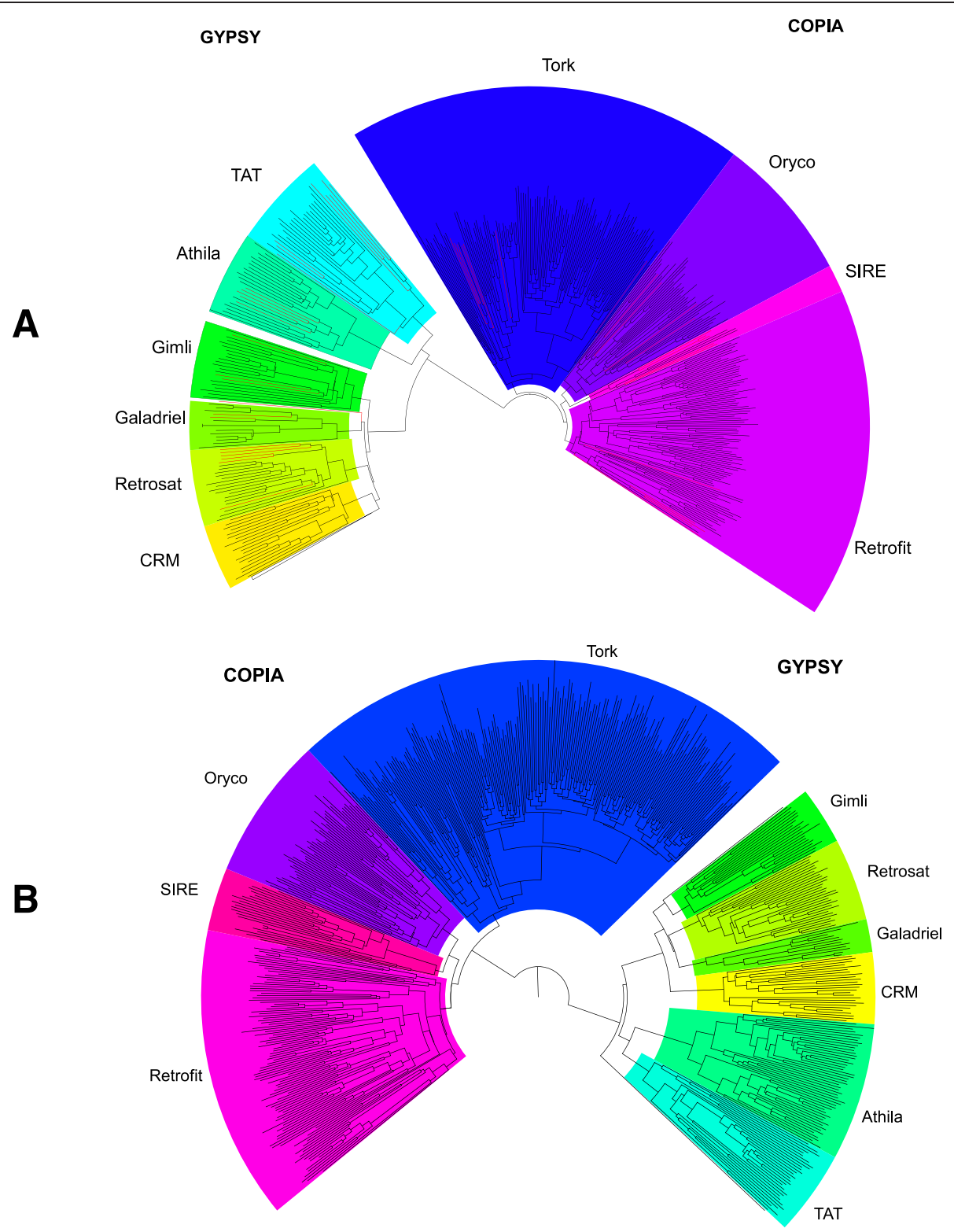

Fig. 2 Phylogenetic analysis of LTR retrotransposons consensus sequences predicted from the E. oleifera (a) and E. guineensis (b) genome sequences. Phylogenetic trees were based on amino-acid alignments of the reverse transcriptase (RT) domains (see Methods for details) The classification was done according the RT reference domains (red lines) downloaded from GyDB [44]

for E guineensis assembly: $39.50 \%(606,458,450 \mathrm{bp})$ and $68.8 \%$, respectively (Fig. 3). In the Eo genome, Class I and Class II consensus TEs mask 32.47 and $4.35 \%$ of the genomic sequences, respectively, whereas the corresponding percentages are 26.8 and $4.08 \%$ for Eg (Fig. 3a). In both genomes, the most abundant among identified TE categories are (in decreasing order): unclassified retrotransposons (RXX; Eo: 13.6 \%; Eg: 10.3 \%), LTR retrotransposons (RLX; Eo: 9.08 \%; Eg: $9.02 \%$ ) and Nonautonomous LTR retrotransposons (RXX-NA; Eo: $7.35 \%$; Eg: $5.5 \%$ ) (Fig. 3b). Taken together, our results suggest that Class I elements form the majority of the TE component in both E. oleifera and E. guineensis genomes, with LTR retrotransposons constituting the largest subclass in both instances.

Among the RLX TE consensus sequences that were previously classified, we further studied the respective contribution of LTR retrotransposons lineages and families to the E. oleifera and E. guineensis genomes. In both $E$. oleifera and E. guineensis genomes, the frequency ratio of Copia to Gypsy retrotransposons is of 5:1. According to our analyses, Tork is the most represented lineage in both genomes (Additional file 3 ) and Sto-4 is largely predominant among families (Additional file 4). 


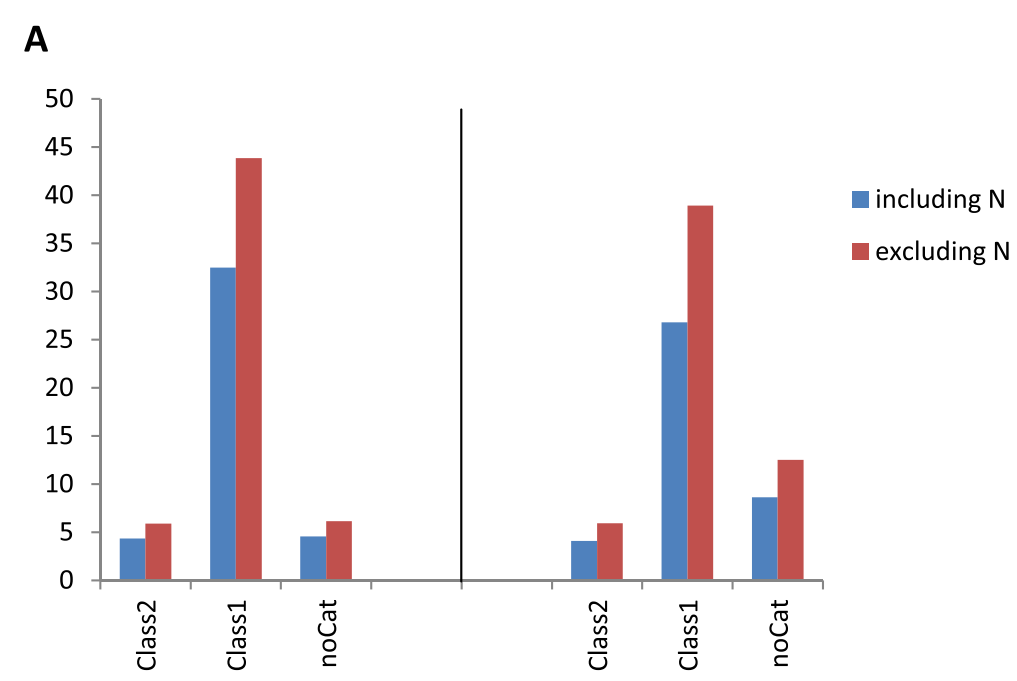

\section{B}

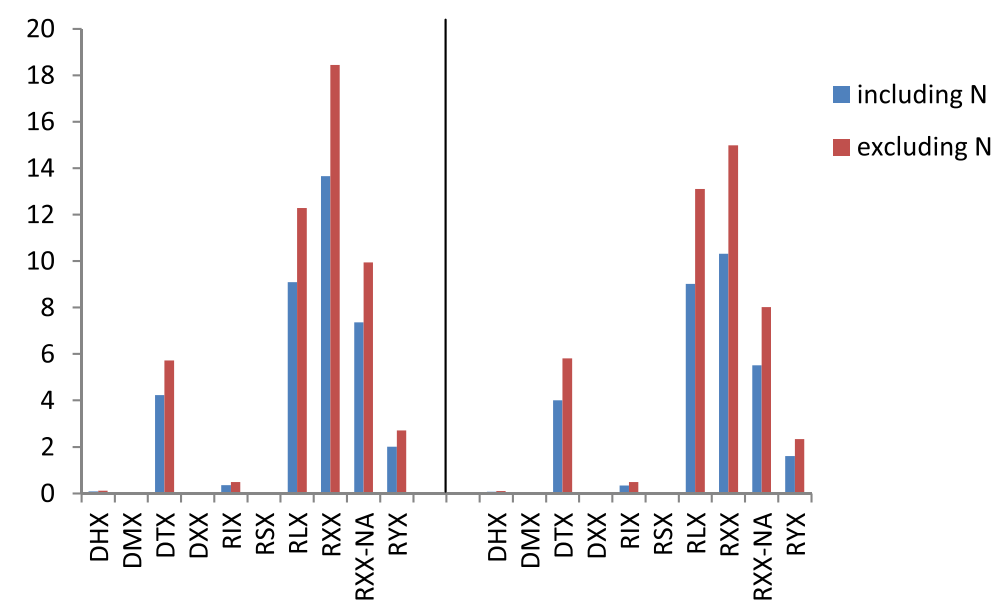

Fig. 3 Percentage of identified TEs in the E. oleifera and in the E. guineensis genomic sequences. a Distribution of the elements among Class I, Class 2 and No Cat (Unclassified), b detailed distribution among the Repeat groups. The percentage of TEs is displayed as found in genomic sequences (in blue) including uncharacterized bases $(N)$ and in genomic sequences excluding ' $N$ ' (in red)

\section{Characterization of full-length LTR retrotransposons in $E$. guineensis}

Since LTR retrotransposons represent the major part of the TE fraction in the E. guineensis genomes, subsequent analyses were focused on this particular class of elements. The RLX consensus sequences previously retrieved from the TEdenovo analysis were found to be incomplete, and therefore we used the LTR_STRUC algorithm [46] in order to identify and localize the corresponding fulllength copies in the genome of E. guineenis.

We collected a total of 583 full-length elements (see their sequences displayed in Additional file 5 and the summary of the full-length elements collection in Table 1). Among them, 241 (41.3\%) were assigned to the Copia superfamily (RLC) and 151 (25.9\%) to the
Gypsy superfamily (RLG). The remaining 191 elements $(32.7 \%)$ could not be classified since no similarity to known Reverse Transcriptase, Integrase nor RNAseH coding domains could be found (Table 1). They were considered hereafter as putative nonautonomous retrotransposons (RXX-NA). However, an interesting point is that 105 of these non-assigned elements included either a putative GAG coding domain alone or both a GAG and a protease (PR) coding domains, suggesting than some of them might belong to the recently discovered subclass of TRGAG elements [47].

As previously, RLC and RLG elements were further classified into lineages. The results obtained with the fulllength LTR retrotransposon elements were essentially 
Table 1 Structural characteristics of the full-length LTR retrotransposons of oil palm E. guineensis

\begin{tabular}{|c|c|c|c|c|c|c|c|}
\hline \multirow[t]{2}{*}{ Superfamily/lineage } & \multirow[t]{2}{*}{ Groups } & \multicolumn{2}{|l|}{ Elements } & \multicolumn{2}{|l|}{ LTR } & \multicolumn{2}{|c|}{ Copies (70-70 \%) } \\
\hline & & Number & $\begin{array}{l}\text { Avg. length (bp) } \\
\text { (min-max) }\end{array}$ & Avg. \% identity & $\begin{array}{l}\text { Avg. length (bp) } \\
\text { (min-max) }\end{array}$ & Number & Genome coverage (\%) \\
\hline \multicolumn{8}{|l|}{ Copia } \\
\hline \multirow[t]{2}{*}{ Oryco } & 25 & 36 & 4796 & 91.85 & 338 & & \\
\hline & & & $4536-5627$ & & $240-469$ & & \\
\hline \multirow[t]{2}{*}{ Sire } & 8 & 8 & 9063 & 89.34 & 1170 & & \\
\hline & & & $4538-11,043$ & & $347-1475$ & & \\
\hline \multirow[t]{2}{*}{ Retrofit } & 74 & 97 & 5075 & 91.62 & 269 & & \\
\hline & & & 2198-9109 & & $83-414$ & & \\
\hline \multirow[t]{2}{*}{ Tork } & 66 & 73 & 7539 & 88.43 & 1069 & & \\
\hline & & & $4118-10,744$ & & $131-248$ & & \\
\hline \multirow[t]{2}{*}{ Undefined clade } & 26 & 27 & 8108 & 89.21 & 1473 & & \\
\hline & & & $1408-10,381$ & & $168-2668$ & & \\
\hline \multirow[t]{2}{*}{ Subtotal } & 199 & 241 & 6252 & 90.34 & 863 & 4816 & 2.32 \\
\hline & & & $1408-11,043$ & & $83-2668$ & & \\
\hline \multicolumn{8}{|l|}{ Gypsy } \\
\hline \multirow[t]{2}{*}{ Athila } & 10 & 10 & 10,438 & 91.07 & 1411 & & \\
\hline & & & $1171-9780$ & & $875-1697$ & & \\
\hline \multirow[t]{2}{*}{ Tat } & 58 & 84 & 10,078 & 90.64 & 666 & & \\
\hline & & & $5572-11,478$ & & $350-1129$ & & \\
\hline \multirow[t]{2}{*}{ CRM } & 16 & 20 & 6455 & 89.51 & 712 & & \\
\hline & & & $8463-5084$ & & $296-1380$ & & \\
\hline \multirow[t]{2}{*}{ Del } & 3 & 3 & 8624 & 86.07 & 1454 & & \\
\hline & & & $6730-10,443$ & & $305-2468$ & & \\
\hline \multirow[t]{2}{*}{ Galadriel } & 3 & 3 & 7463 & 90.83 & 1777 & & \\
\hline & & & $5826-10,130$ & & $533-3861$ & & \\
\hline \multirow[t]{2}{*}{ G-Rhodo } & 1 & 1 & 5506 & 90.40 & 448 & & \\
\hline & & & & & $447-450$ & & \\
\hline \multirow[t]{2}{*}{ Reina } & 15 & 16 & 5921 & 91.62 & 607 & & \\
\hline & & & $5085-10,286$ & & $266-2490$ & & \\
\hline \multirow[t]{2}{*}{ Undefined clade } & 13 & 14 & 6561 & 88.07 & 824 & & \\
\hline & & & $2113-11,900$ & & $110-1413$ & & \\
\hline \multirow[t]{2}{*}{ Subtotal } & 119 & 151 & 8744 & 90.30 & 987 & 1934 & 1.06 \\
\hline & & & $2113-11,900$ & & $110-3861$ & & \\
\hline \multirow[t]{2}{*}{ Putative non autonomous (RXX-NA) } & 174 & 191 & 4911 & 88.20 & 828 & 3804 & 1.01 \\
\hline & & & $1279-11,793$ & & $83-5259$ & & \\
\hline \multirow[t]{2}{*}{ Total } & 492 & 583 & 6458 & 89.63 & 753 & 10,554 & 4.39 \\
\hline & & & $1279-11,900$ & & $83-5259$ & & \\
\hline
\end{tabular}

identical to those previously described with the partial TE consensus sequences (Additional file 6).

Further examination of the internal ORFs revealed that the majority ( $83 \%$ ) of the full-length RLC and RLG elements encode either four (108 elements) or five (215 elements) protein coding domains (Additional file 7A). In accordance with current standards of TE classification
[1], the elements belonging to the latter category (which represent $55 \%$ of total full-length LTR retrotransposons) contain both the POL and the GAG coding regions that are required for transposition. They are therefore susceptible to include autonomous elements, however further sequence analyses show that these domains are most likely non-functional due to frameshifts and 
mutations. Also, we observe that the frequency of each protein coding domain is similar between both retrotransposons super-families, and that over $90 \%$ of the elements include all three RT, INT and RH coding domains regardless of the superfamily (Additional file 7B). By contrast, LTR length is extremely variable, ranging from 83 to 5259 bp with an average value of 753 bp (Table 1). Nevertheless, within most lineages the average LTR length of oil palm retrotransposons is in agreement with data collected from other plant species.

\section{Full-length LTR retrotransposon copy number and chromosomal distribution}

In order to cluster the 583 full-length LTR retrotransposons of oil palm into families based on sequence relatedness, we eliminated sequence redundancy according to the recommendations of Wicker et al. [1], i.e. elements are deemed related if a sequence identity of at least $80 \%$ is detected across $80 \%$ of the length of the retrotransposon. However, due to its high level of stringency and, possibly, to the structural diversity of LTR retrotransposons in the oil palm genome, this analysis was unable to detect related elements in our case (results not shown). An empirically determined threshold of $70 \%$ (of sequence identity) $-70 \%$ (of sequence length) was finally used and enabled the identification of 492 groups, including 199 Copia, 119 Gypsy and 174 putative non-autonomous retrotransposons (RXX-NA), as potential TE families (Table 1). Most of these groups include a single element, further strengthening the hypothesis of a high level of sequence diversity of LTR retrotransposons in oil palm. However, our analysis is based on the current release of the E. guineenis genome [39] and an improvement of both the overall quality of genomic sequences and scaffold size of the assembly are necessary before this assumption can be confirmed.

The number of full-length LTR retrotransposon copies in the E. guineenis genome was estimated using one reference element from each of the 492 groups. However, because of the high level of sequence diversity among these elements, it was not possible to assign each copy to a single reference sequence unequivocally, and as a result copy number was determined globally for each superfamily.

A total of 10,554 full-length copies were detected, representing $4.39 \%$ of the oil palm genome assembly (Table 1). Copies belonging to the Copia superfamily (4816) displayed the highest rate of genome coverage (approximately $2.32 \%$ ), whereas the Gypsy superfamily (1934 copies) accounted for $1.06 \%$.

To gain insight into possible correlations between the respective distribution of predicted coding sequences and full-length LTR retrotransposons, we plotted the LTR retrotransposon density along the 16 E. guineensis pseudo-chromosome sequences accounting for $43 \%$ of the whole genome assembly [39]. Among the 10,554 fulllength LTR retrotransposon copies identified previously, $5703(\sim 54 \%)$ could be mapped to the 16 assembled pseudo-chromosomes (Fig. 4). The average LTR retrotransposon density was 8.75 sequences per $\mathrm{Mb}$ with 3.43 and 1.96 respectively for Copia and Gypsy. In addition, the analysis of TE distribution with respect to predicted genes showed that full-length Gypsy elements were distributed uniformly across the 16 pseudo-chromosomes, irrespective of gene location, whereas a highly significant negative correlation was observed between the density of full-length Copia elements and gene density $(R=-0.46, P=0.0000)$ (Additional file 8). This latter result seems to indicate a higher abundance of Copia in gene-poor regions compared to gene-rich regions in the E. guineensis genome.

\section{Transcriptional activity of full-length LTR retrotransposons}

The relative expression of the 583 full-length LTR retrotransposons was assessed through the computational analysis of publicly available RNAseq libraries from eight different oil palm tissues (see Methods). Overall, most of the elements show a low level of transcription regardless of the tissue, whereas 63 retrotransposons are expressed in at least one of the studied tissues. Among these, most are transcribed in a limited number of tissues with no significant clustering (Fig. 5). It is interesting to note that elements classified as potential non-autonomous LTR retrotransposons (RXX-NA, Table 1) account for $57.8 \%$ of the overall transcriptional activity related to these 63 LTR retrotransposons in the eight tissues studied, $v s$. $19.3 \%$ and $22.8 \%$ for Copia and Gypsy elements, respectively (Additional file 9A). Moreover, when comparing expression levels related to these 63 elements between tissues, we observe that it is the highest in shoot apices (18.2\% of the cumulated expression of these elements in all eight libraries, Additional file 9B) and the lowest in young female flowers (6.3\%). Intriguingly, this share is increased to $13.4 \%$ in more mature female flowers, mostly due to the upregulation of Tork elements and, to a lesser extent, that of Tat elements which translate into 62- and 4-fold increases in count numbers fort these lineages between both inflorescence stages, respectively. Only one element (Eg5-3661-PTB13-L60-145; RXX-NA) shows transcriptional activity in all tested tissues, whereas the Eg5-4398-B2-L43-392 (RXX-NA) element generates the strongest expression signal observed in this study, in the shoot apex.

\section{Putative insertion time and evolution of LTR} retrotransposon populations in the $E$. guineensis genome In order to infer the evolutionary history of LTR retrotransposon populations in the oil palm genome, we 


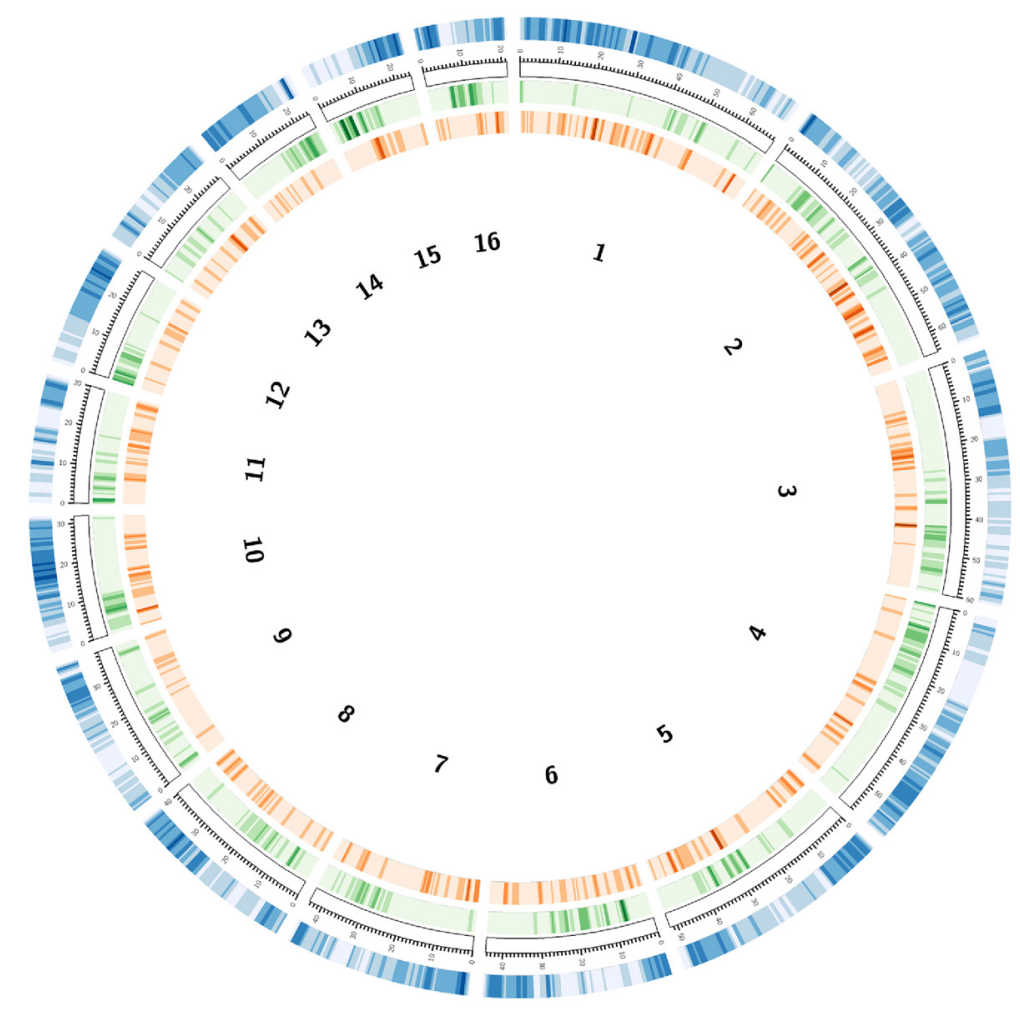

Fig. 4 Chromosomal distribution full-length LTR retrotransposons in the Elaeis guineensis genome. Green track: Copia elements; orange track: Gypsy elements; blue track: predicted genes. The intensity of the coloring is directly proportional to the respective sequence densities

evaluated sequence divergence between the 5' and 3' LTRs of each full-length element. Because of the requirements of the transposition mechanism, both LTR sequences of a single retrotransposon are $100 \%$ identical at the time of its insertion into the genome. Through time, they progressively diverge from one another by accumulating mutations, such as nucleotide substitutions. It is therefore possible to calculate the nucleotide substitution rate between both LTRs in order to roughly discriminate the respective insertion times of different retrotransposon populations [48].

Our results, illustrated in Fig. 6, suggest that the oil palm genome underwent several waves of LTR retrotransposon amplification events, with different temporal patterns of transpositional activity for the main superfamilies Copia and Gypsy. Massive insertion events of Copia retrotransposons into the oil palm genome were displayed in two distinct peaks. By contrast, we observe a single peak for the insertion of Gypsy elements and Non-Autonomous LTR retrotransposons. Finally, we detect very few recent insertion events in our dataset, with the notable exception of the Pi519857_RT4_B11_L9_394 element (Copia superfamily, Oryco lineage).

\section{Discussion}

In the original paper describing both oil palm genomes [39], the repeat content of E. guineensis had been estimated to approximately $57 \%$ of its $1.8 \mathrm{~Gb}$ genome with a large prevalence of LTR retrotransposons, while no such analysis had been performed in E. oleifera. Thus, to our knowledge the present study constitutes the first attempt to compare TE populations between both oil palm genomes and to further characterize LTR retrotransposon families and lineages in the major oil crop E. guineensis. In other plant species, genome-wide analyses of TE populations have not only provided clues on the individual history of each genome but they have also yielded useful information about the origins of modern-days crop genomes through domestication, speciation and hybridization. In wheat, such studies have demonstrated the occurrence of differential dynamics of TE proliferation between the $\mathrm{A}$ and $\mathrm{B}$ subgenomes, both prior and after allotetraploidization [49]. In rice, the comparison of the transpositional history of LTR retrotransposons between the Japonica and Indica sub-species has provided evidence for two independent domestication processes in Asian rice [50].

As a first step in our study, we have annotated the transposable elements from both Elaeis genomes through a de novo approach [42]. While we found that the overall repeat content is nearly identical between both genomes, the comparison between the two TE databases revealed the occurrence of 6 times more Unclassified Repeats in 


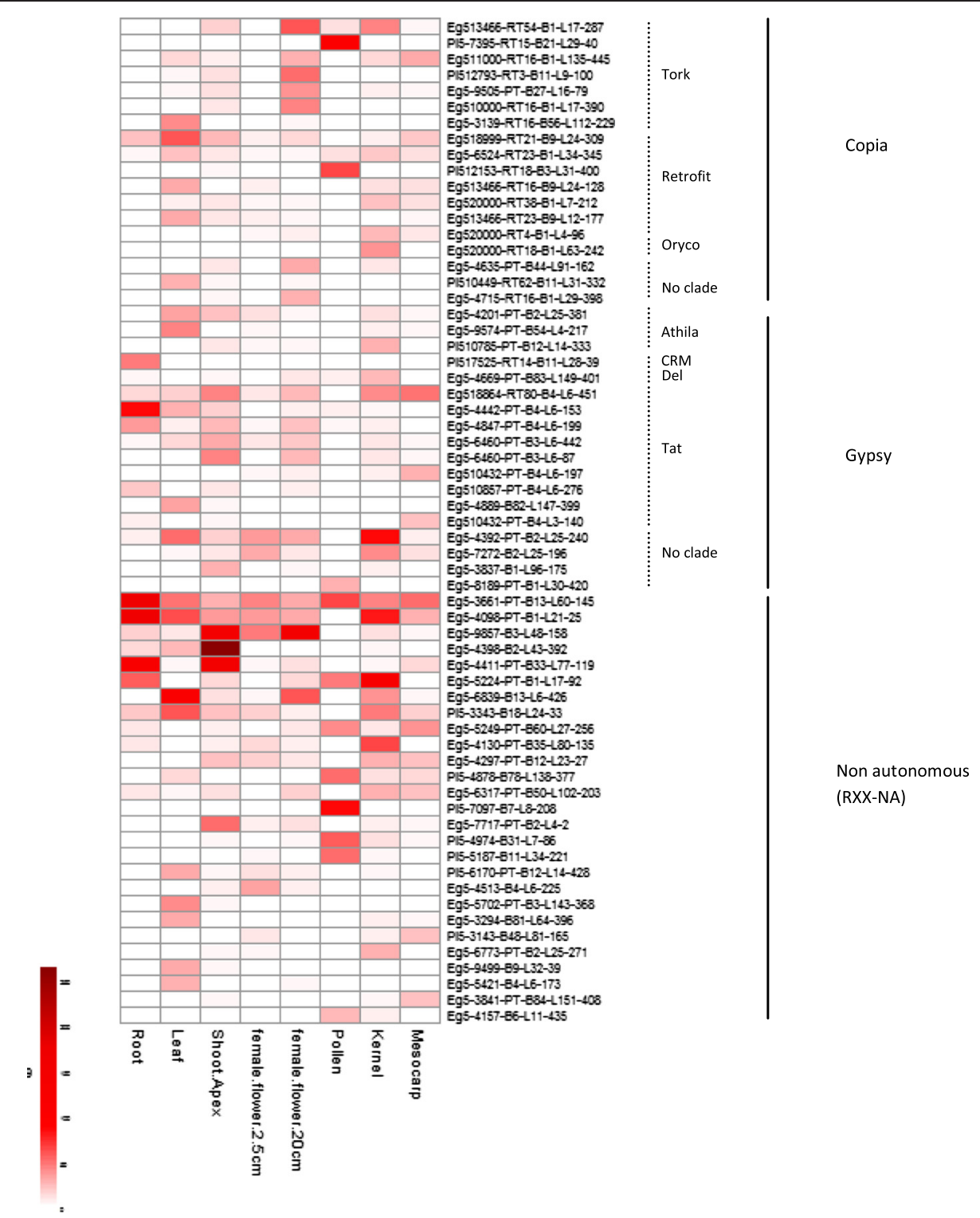

Fig. 5 Expression map of full-length LTR retrotransposons in Elaeis guineensis tissues. The intensity of the coloring is directly proportional to the relative expression of the retrotransposons (see text for details)

the E guineensis genome compared to E. oleifera. This discrepancy in the distribution of TE categories is most likely due to differences in both sequencing completion and assembly quality between both draft genomes, resulting in a lesser coverage of this TE subclass in E. oleifera. However, the distribution of all other TE categories was similar between the E. guineensis and E. oleifera genomes, with a large prevalence of Class I elements. Furthermore, among the LTR retrotransposons that form the major part of the TE fraction, we discovered a comparable diversity within the Gypsy and Copia super-families between both oil palm genomes. Our results also highlighted a remarkable expansion of the same retrotransposon lineages (namely Retrofit and Tork), indicating that they were intensively active during the evolution of both palm genomes. In both species, Copia elements are more abundant than Gypsy and one LTR retrotransposon family, named Sto- 4 and belonging to the Copia superfamily, is the most represented. This result is consistent with a previous analysis of 32 Reverse Transcriptase coding sequences, from which it was extrapolated that Copia elements might make up about $6 \%$ of the E. guineensis genome [37]. Also, our findings are in accordance with those from Singh and coworkers [39] since they observed a significant expansion of a RIRE-like member of the Sto-4 family in the E. guineensis genome. An interesting point is that the RIRE1 element has originally been identified in $O$. australiensis, a wild relative of rice [51], where its 30,000 copies represent 


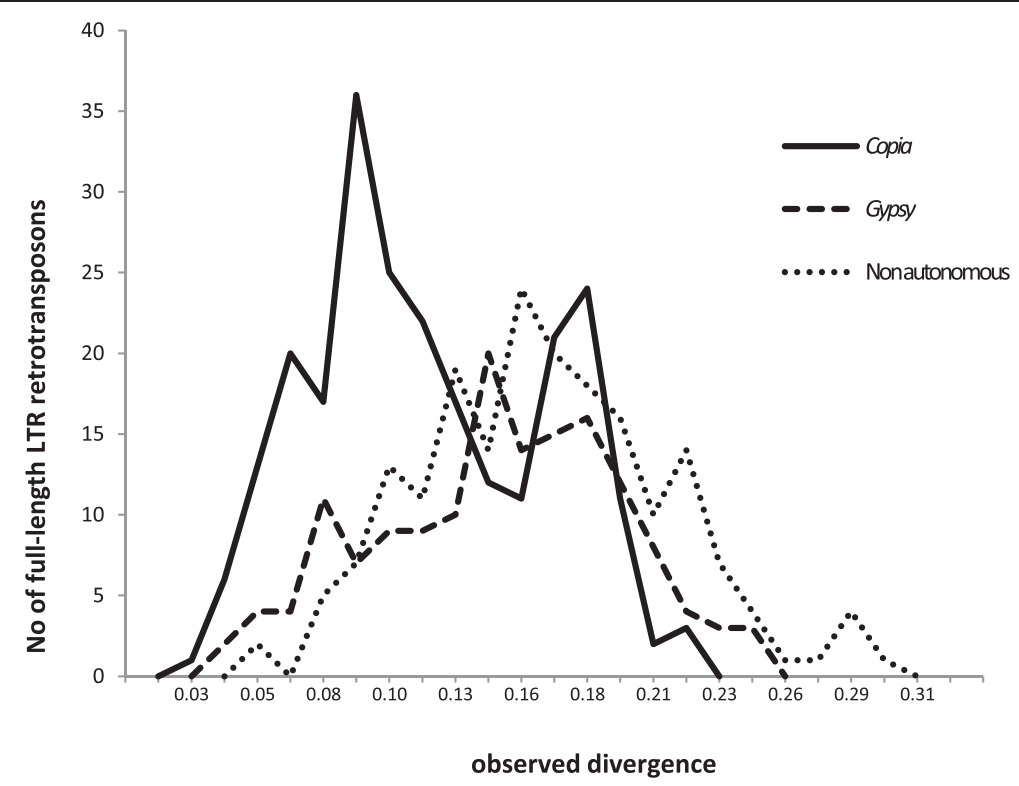

Fig. 6 Estimated insertion of full-length LTR retrotransposons into the oil E. guineensis genome. The horizontal axis represents sequence divergence; see text for details

$27 \%$ of the host genome. The extreme similarity in LTR retrotransposons distribution between both Elaeis genomes makes it tempting to hypothesize that the massive amplification of some families, especially those belonging to the Tork lineage, occurred before both palm genomes diverged from each other. According to some authors, this divergence took place 51 Mya ago [39] but other studies using different datasets and methods suggested that the speciation event might be much more recent: between 7 Mya ago [52] and 15 Mya ago according to Baker and Couvreur [53]. Clearly, more in-depth phylogenetic analyses of the oil palm genomes are needed to ascertain both the time frame of this event and the dating of TE insertions since the genomic organization of TE populations results from a dynamic balance between amplification bursts and recombination events leading to DNA loss $[1,6,54,55]$.

It must be kept in mind that the approach that were used here, and which are based on the de novo reconstruction of TE consensus sequences from available genome contig sequences, may have led to an underestimation of the repeat content. In any case, the present study represents a first step towards the construction of a comprehensive oil palm TE catalogue.

Since LTR retrotransposons represent the vast majority of the Transposable Elements found in oil palm genomes, we have further characterized full-length LTR retrotransposons in the E. guineensis genome. Interestingly, when analyzing retrotransposon density across the genome, we find a preferential insertion of full-length Copia elements in relatively gene-poor regions of the assembled pseudo-chromosomes, whereas the Gypsy elements appear to be randomly distributed. In oil palm, previous in situ hybridization experiments performed by Castilho et al. [36] led to a similar conclusion about the Copia elements, and Schnable et al. [3] also observed such a differential distribution of both super-families in maize. Comparisons performed between partially or completely sequenced plant genomes have shown that LTR elements are mostly concentrated in gene-poor regions, with variations according to superfamily or lineage [56]. In relatively gene-rich regions however, the dispersion of LTR retrotransposons appears to be greater within small plant genomes $(<500 \mathrm{Mb})$ as opposed to large ones, where they occur as stretches of nested elements [56].

We find that, although most of the elements identified in the E. guineensis genome include the protein coding domains that are required for transposition, the sequences are disrupted by premature stop codons or frameshifts, indicating that they might no longer be functional. Furthermore, the analysis of the sister LTRs from each of these retrotransposons failed to detect any element displaying $100 \%$ nucleotide identity between these repeats, which is a hallmark of recently inserted TEs. Taken together with estimates of LTR sequence divergence and the high degree of structural diversification observed between retrotransposon lineages, this result suggests that no significant insertion event occurred recently in the African oil palm genome. However, in the absence of data on base substitution rates in oil palm genes and TEs, it is not possible to improve on the 
dating of these insertion events through the use of a "molecular clock" such as was used in the rice genome [57]. Despite this limitation, our analysis shows that the full-length Copia and Gypsy elements studied were inserted into the oil palm genome around the same period of time but exhibited distinct dynamics. Interestingly, no recent insertion event was inferred, further strengthening the hypothesis that the LTR retrotransposons of the oil palm genome are most likely transpositionally inactive. This conclusion is in contrast to observations made in other plant genomes such as maize [58], rice $[55,57]$ and coffee [59], in which recent retrotransposon insertion events have been detected but quite similar to the situation in the banana [60] and olive tree genomes [61]. In the latter genome, transpositional activity from LTR retrotransposons has been shown to be decreasing over time but active copies are nevertheless still detectable.

These various clues to the lack of transpositional activity from the LTR retrotransposons analyzed, as well as the high proportion of retrotransposons classified as non-autonomous in our study such as LARD [62] and TRIM [63], paint a picture of the E. guineensis genome as a landscape where TEs are mostly, if not completely, immobile. However, the presence in the genome of trancriptionally and/or transpositionally active copies, enabling the trans-complementation of these presumably inactive elements, cannot be excluded at this stage. Indeed, our investigation of the transcriptional activity of our full-length LTR retrotransposons shows that some of them are expressed, albeit mostly at low levels, in different oil palm tissues even though their transcriptional and/or their transpositional autonomy is most likely impaired because of the accumulated mutations. Our analysis also shows that the expression levels of these transcriptionally active retrotransposons are highly variable between both lineages and tissues. We hypothesize that this activity could be related to the Developmental Relaxation of TE silencing (DRTS) [64] that has been shown to result in relatively elevated TE expression, notably in shoot and inflorescence meristems of both maize and rice [65-67]. Although the exact role of this phenomenon is yet to be elucidated, it has been proposed to contribute to the reinforcement of small RNAs-mediated TE silencing through their transient derepression in specific tissues, as well as to the epigenetic regulation of both genes and genome in connection with cell specification and plant development [64]. Overall, these data suggest that these elements could still have the potential to interfere with the expression of neighboring genes, through either the production of readthrough or antisense transcripts [10] or the alteration of epigenetic marks $[68,69]$, and lead to phenotypic variations. Our group has recently demonstrated that the splicing of the EgDEF1 gene, which is believed to be involved in the mantled floral phenotype, is strongly affected in variant flowers, possibly as a consequence of the intronic insertion of an inactive Gypsy retrotransposon [70]. Additional work will be required to further explore the interactions between the mechanisms regulating genes and $\mathrm{TE}$ expression in the oil palm genome.

\section{Conclusions}

The present study presents the most comprehensive description of oil palm LTR retrotransposons to date. Our results, which reveal a congruence in the tranpositional history of LTR retrotransposons between E. oleifera and $E$. guineensis, will provide crucial information for dating their divergence and further, to elucidate the history of genome evolution in the Arecaceae palm family. Moreover, our TE database will be a helpful resource in future studies aiming at assessing the possible contribution of LTR retrotransposons to genome and transcriptome variations resulting from the in vitro somatic embryogenesis process, especially in the context of the oil palm mantled phenotype.

\section{Methods}

\section{Data source}

The oil palm Elaeis guineensis and Elaeis oleifera genome sequences generated by Malaysian Oil Palm Genome Programme (MyOPGP) [39] have been downloaded from the NCBI and MPOB web sites (http://www.ncbi.nlm.nih. gov/assembly/GCA_000441515.1/; http://genomsawit.mpob.gov.my/genomsawit/, in August 2013; P5-build and EG5-linked assemblies for E. guineensis and O8-build for E. oleifera).

\section{Annotation of transposable elements}

A combination of manual approaches and automated programs (REPET package V.2.2-RC; [42]) were used to identify, classify and annotate repeated sequences from the largest scaffolds (size $>300 \mathrm{kbp}$ ) assembled for each of the studied genomes. The sequences that were investigated include 991 scaffolds amounting to a total of $730,618,412$ bp of genome sequence from the O8-build and 846 scaffolds representing 1,068,102,326 bp from the P5-build, respectively. TE consensus nucleotide sequences were classified according to the Repbase database [43] and named according to the classification proposed by Wicker et al. [1]: DHX (Helitron), DMX (Maverick), DTX (TIR Transposon), DXX (MITE) for Class II elements, and RIX (LINE), RLX (LTR Retrotransposon), RSX (SINE), RXX (unclassified or nonautonomous retrotransposons), RYX (DIRS) for Class I element. Consensus sequences assigned as LTR retrotransposons were further classified through the phylogenetic analysis of their reverse transcriptase (RT) amino-acid domains: putative RT coding domains were 
first identified in consensus nucleotide sequences using BLASTX [71] and translated using Genewise [72], then the resulting RT amino acid sequences (with a minimum length of 150 residues) and reference RTs from Gypsy Database 2.0 [44] were aligned with ClustalW to construct a NJ tree that was finally edited with FigTree (http://tree.bio.ed.ac.uk/software/figtree/). Repeatmasker [45] was used with default parameters so that sequences with less than $80 \%$ identity to the reference sequence were masked.

The LTR_STRUC 1.1 algorithm [46] was used with default parameters in order to detect full-length LTR retrotransposons among the complete dataset of Elaeis guineensis scaffolds $(1,535,150,282 \mathrm{bp})$. The following structural definition was used for the full-length LTR retrotransposon, regardless of its transcriptional or transpositional ability: a repeat element that i) is delimited by highly similar 5' and 3' LTRs; ii) has generated a Target Site Duplication (TSD) on each border of the insertion site into the host genome; iii) includes putative primer binding site (PBS) and polypurine tract (PPT) sequences at the 5' and 3' the of its internal sequence, respectively.

\section{Annotation and phylogenetic analysis of full-length LTR retrotransposons}

Raw results from the LTR_STRUC detection were analyzed as follow to construct an oil palm full-length LTR retrotransposons reference library. This data was classified into Gypsy (RLG) and Copia (RLC) superfamilies according to their similarity with the content of the GyDB domain libraries [44] and the occurrence and respective location of the protein coding domains for the Reverse-transcriptase (RT), Integrase (INT) and RNAseH (BLASTX E-value cut-off: 1e-4). Sequences were classified into the RXX category if only the GAG (capsid) and PR (protease) coding domains, or the GAG alone, were detected, or if no sequence similarity could be found. Predicted sequences larger than $12 \mathrm{~kb}$ were not retained for further analysis. Classification of predicted RLG and RLC full-length LTR retrotransposons was confirmed and completed by phylogenetic analyses as described previously, using four previously published oil palm RT domains (GenBank accessions AJ507412 to AJ507415 [38]).

\section{Copy number estimation, distribution and insertion time}

All identified full-length LTR retrotransposons were clustered into families or group using the CD-HIT software [73] with a minimum of $70 \%$ of nucleotide identity and a minimum sequence coverage of $70 \%$ between related elements. Within each family, the longest sequence displaying a high percentage of nucleotide sequence identity between both LTR regions was selected as the reference sequence. The copy number of each superfamily was determined using Censor [74]. A copy is considered as complete if it covers a minimum of $70 \%$ of the reference sequence with a minimum of $70 \%$ of nucleotide identity. The density of retrotransposon distribution along pseudo-chromosomes was calculated using a home-made shell script, with a $1 \mathrm{Mbp}$ sliding window (step size of $500 \mathrm{kbp}$ ) and plotted using CIRCOS [75].

The insertion times of the previously identified fulllength LTR retrotransposons were estimated based on the sequence divergence between the 5' and 3' LTR of each element, as determined through successively aligning the sequences using Stretcher then implementing the Kimura 2-parameter method in Distmat (EMBOSS package). An average base substitution rate of 1.3E-8 was used in accordance with Ma and Bennetzen [57].

\section{Transcriptional analysis of LTR retrotransposons}

The transcriptional analysis was carried out using data deposited into NCBI's databases (Bioproject number PRJNA201497). Eight sets of oil palm (Elaeis guineensis) transcriptome data from different tissues were reanalyzed: root (SRX278062), leaf (SRX278048), shoot apex (SRX278055), young female flower (SRX278052), mature female flower (SRX278053), pollen (SRX278051), kernel (SRX278018) and mesocarp (SRX278017). Data quality was evaluated with FastQC [76] and low quality reads were excluded with Cutadapt [77]. Reads were mapped against our full-length LTR retrotransposons reference library using the BWA-MEM package with default parameters [78]. Samtools [79] was used to calculate the number of mapped reads (counts) for each reference sequence and normalization was performed using the EdgeR package [80]. The graphical representation of full-length LTR-retrotransposons expression in the different tissues was generated by the pheatmap $R$ package [81].

\section{Availability of data and materilas}

The data sets supporting the results of this article are included within the article and its additional files.

\section{Additional files}

Additional file 1: De novo consensus TE databases. Contains the $E$. oleifera (E08_denovoLibTEs.txt) and E. guineensis (p5_denovoLibTEs.txt) databases. (ZIP 15,038 kb)

Additional file 2: Summary of the contents of the $E$. oleifera and $E$. guineensis TE databases. (PDF $9 \mathrm{~kb}$ )

Additional file 3: Sequence coverage of LTR retrotransposon lineages in the E. oleifera (Eo) and in E. guineensis $(\mathrm{Eg})$ genomes. (PDF $35 \mathrm{~kb}$ )

Additional file 4: Sequence coverage of LTR retrotransposon families in the E. oleifera (Eo) and in E. guineensis (Eg) genomes. (PDF $356 \mathrm{~kb}$ ) 
Additional file 5: Overview of LTR_STRUC results. For each full-length LTR retrotransposon, superfamily, putative lineage, overall length, identity percentage between both LTR sequences, target site duplication (TSD), polypurine tract (PPT) and tRNA binding site are provided, as well as the sequences of the element and of its LTRs. (XLSX $1543 \mathrm{~kb}$ )

\section{Additional file 6: Phylogenetic analysis of full-length LTR retrotransposon sequences predicted from the E. guineensis genome. See Methods for details. (PDF $332 \mathrm{~kb}$ )}

Additional file 7: Protein coding domain composition of full-length LTR retrotransposons of oil palm E. guineensis. A: Number of protein coding domain identified per retrotransposon (figures correspond to the number of elements displaying either 1, 2, 3, 4 or 5 domains). B: Frequency of the different protein coding domain in the Copia and Gypsy superfamilies. $\mathrm{AP}=$ protease, $\mathrm{INT}=$ integrase, $\mathrm{RT}=$ reverse transcriptase, $\mathrm{RH}=$ ribonuclease, $\mathrm{GAG}=$ capsid. (PDF $174 \mathrm{~kb}$ )

Additional file 8: Relationship between the density (sequences per $\mathrm{Mb}$ ) across chromosomes of predicted coding sequences and that of Copia (A) and Gypsy (B) full-length elements. (PDF $153 \mathrm{~kb}$ )

Additional file 9: Transcriptional activity of 63 LTR retrotransposons. A: Percentage of normalized read counts per superfamily. B: Percentage of normalized read counts per tissue. (PDF $178 \mathrm{~kb}$ )

\section{Abbreviations}

DIRS: Dictyostelium intermediate repeat sequence; LARD: Large retrotransposon derivative; LINE: Long interspersed nuclear element; LTR: Long terminal repeat; MITE: Miniature inverted-repeat transposable element; SINE: Short interspersed nuclear element; TIR: Terminal inverted repeat; TRIM: Terminal-repeat retrotransposon in miniature.

\section{Competing interests}

The authors declare that they have no competing interests.

\section{Authors' contributions}

TB and RG designed the study. TB, MTDA and RG performed sequence analyses. TB and RG interpreted the results. SD performed the statistical analysis. TB, EJ and RG drafted the manuscript. All authors read and approved the final manuscript.

\section{Acknowledgements}

MTDA was funded by the "FloriPalm" Specific Research Agreement between CIRAD and FASSB and the "PalMarkers" research contract between CIRAD and PalmElit SAS.

The funding bodies played no role in the design, collection, analysis, and interpretation of data; in the writing of the manuscript; nor in the decision to submit the manuscript for publication.

\section{Author details}

${ }^{1}$ CIRAD, UMR DIADE (IRD, UM), 34394 Montpellier, France. ${ }^{2}$ IRD, UMR DIADE (IRD, UM), 34394 Montpellier, France. ${ }^{3}$ IRD, UMR IPME (IRD, CIRAD, UM), 34394 Montpellier, France.

Received: 23 June 2015 Accepted: 7 October 2015

\section{Published online: 15 October 2015}

\section{References}

1. Wicker T, Sabot F, Hua-Van A, Bennetzen JL, Capy P, Chalhoub B, et al. A unified classification system for eukaryotic transposable elements. Nat Rev Genet. 2007;8:973-82.

2. The Arabidopsis Genome Initiative. Analysis of the genome sequence of the flowering plant Arabidopsis thaliana. Nature. 2000;408:796-815.

3. Schnable PS, Ware D, Fulton RS, Stein JC, Wei F, Pasternak S, et al. The B73 maize genome: complexity, diversity, and dynamics. Science. 2009;326:1112-5.

4. Brenchley R, Spannagl M, Pfeifer M, Barker GLA, D'Amore R, Allen AM, et al. Analysis of the bread wheat genome using whole-genome shotgun sequencing. Nature. 2012;491:705-10.

5. The International Barley Genome Sequencing Consortium. A physical, genetic and functional sequence assembly of the barley genome. Nature. 2012;491:711-6
6. Bennetzen $J \mathrm{~L}, \mathrm{Ma} J$, Devos KM. Mechanisms of recent genome size variation in flowering plants. Ann Bot. 2005;95:127-32.

7. Morgante M, De Paoli E, Radovic S. Transposable elements and the plant pan-genomes. Curr Opin Plant Biol. 2007;10:149-55 [Genome Studies and Molecular Genetics / Edited by Stefan Jansson and Edward S Buckler]

8. Tenaillon MI, Hollister JD, Gaut BS. A triptych of the evolution of plant transposable elements. Trends Plant Sci. 2010;15:471-8.

9. Kumar A, Bennetzen JL. Plant retrotransposons. Annu Rev Genet. 1999;33:479-532.

10. Lisch D. How important are transposons for plant evolution? Nat Rev Genet. 2013;14:49-61.

11. Miller WJ, Capy P. Applying mobile genetic elements for genome analysis and evolution. Mol Biotechnol. 2006;33:161-74.

12. Todorovska E. Retrotransposons and their role in plant-genome evolution. Biotechnol Biotechnol Equip. 2007;21:294-305.

13. Peterson-Burch BD, Nettleton D, Voytas DF. Genomic neighborhoods for Arabidopsis retrotransposons: a role for targeted integration in the distribution of the Metaviridae. Genome Biol. 2004;5:R78.

14. Jin W, Melo JR, Nagaki K, Talbert PB, Henikoff S, Dawe RK, et al. Maize centromeres: organization and functional adaptation in the genetic background of oat. Plant Cell. 2004;16:571-81.

15. Casacuberta E, González J. The impact of transposable elements in environmental adaptation. Mol Ecol. 2013;22:1503-17.

16. Pélissier T, Mathieu O. Glue for jumping elements: epigenetic means for controlling transposable elements in plants. In: Grandbastien M-A, Casacuberta JM, editors. Plant transposable elements. Berlin Heidelberg: Springer; 2012. p. 125-45 [Topics in Current Genetics, vol. 24]

17. von Sternberg R, Shapiro JA. How repeated retroelements format genome function. Cytogenet Genome Res. 2005;110:108-16.

18. Hoen DR, Bureau TE. Transposable element exaptation in plants. In: Grandbastien M-A, Casacuberta JM, editors. Plant transposable elements. Berlin Heidelberg: Springer; 2012. p. 219-51 [Topics in Current Genetics, vol. 24]

19. Feschotte $\mathrm{C}$. The contribution of transposable elements to the evolution of regulatory networks. Nat Rev Genet. 2008;9:397-405.

20. Cui X, Cao X. Epigenetic regulation and functional exaptation of transposable elements in higher plants. Curr Opin Plant Biol. 2014;21:83-8 [SI: Cell Signalling and Gene Regulation]

21. Grandbastien M-A. Activation of plant retrotransposons under stress conditions. Trends Plant Sci. 1998;3:181-7.

22. Bui QT, Grandbastien M-A. LTR retrotransposons as controlling elements of genome response to stress? In: Grandbastien M-A, Casacuberta JM, editors. Plant transposable elements. Berlin Heidelberg: Springer; 2012. p. 273-96 [Topics in Current Genetics, vol. 24]

23. Makarevitch I, Waters AJ, West PT, Stitzer M, Hirsch CN, Ross-lbarra J, et al. Transposable elements contribute to activation of maize genes in response to abiotic stress. PLoS Genet. 2015;11:e1004915.

24. Paszkowski J. Controlled activation of retrotransposition for plant breeding. Curr Opin Biotechnol. 2015;32:200-6 [Food Biotechnology - Plant Biotechnology]

25. Hirochika H, Sugimoto K, Otsuki Y, Tsugawa H, Kanda M. Retrotransposons of rice involved in mutations induced by tissue culture. Proc Natl Acad Sci. 1996;93:7783-8.

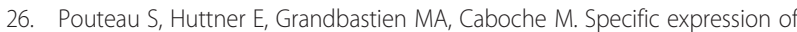
the tobacco Tnt1 retrotransposon in protoplasts. EMBO J. 1991;10:1911-8.

27. Hirochika $\mathrm{H}$. Activation of tobacco retrotransposons during tissue culture. EMBO J. 1993;12:2521-8

28. Suoniemi A, Narvanto A, Schulman AH. The BARE-1 retrotransposon is transcribed in barley from an LTR promoter active in transient assays. Plant Mol Biol. 1996;31:295-306.

29. Kaeppler SM, Kaeppler HF, Rhee Y. Epigenetic aspects of somaclonal variation in plants. Plant Mol Biol. 2000;43:179-88.

30. Smulders M, de Klerk G. Epigenetics in plant tissue culture. Plant Growth Regul. 2011;63:137-46.

31. Miyao A, Nakagome $M$, Ohnuma $T$, Yamagata $H$, Kanamori $H$, Katayose $Y$ et al. Molecular spectrum of somaclonal variation in regenerated rice revealed by whole-genome sequencing. Plant Cell Physiol. 2012;53:256-64.

32. Corley RHV, Lee CH, Law IM, Wong CY. Abnormal flower development in oil palm clones. Planter. 1986;62:233-40.

33. Jaligot E, Rival A, Beulé T, Dussert S, Verdeil JL. Somaclonal variation in oil palm (Elaeis guineensis Jacq.): the DNA methylation hypothesis. Plant Cell Rep. 2000;19:684-90. 
34. Jaligot E, Beulé T, Rival A. Methylation-sensitive RFLPs: characterisation of two oil palm markers showing somaclonal variation-associated polymorphism. Theor Appl Genet. 2002;104:1263-9.

35. Jaligot E, Beulé T, Baurens FC, Billotte N, Rival A. Search for methylationsensitive amplification polymorphisms associated with the "mantled" variant phenotype in oil palm (Elaeis guineensis Jacq.). Genome. 2004;47:224-8.

36. Castilho A, Vershinin A, Heslop-Harrison JS. Repetitive DNA and the chromosomes in the genome of oil palm (Elaeis guineensis). Ann Bot. 2000;85:837-44.

37. Price Z, Dumortier FD, MacDonald D, Mayes S. Characterisation of copia -like retrotransposons in oil palm (Elaeis guineensis Jacq.). Theor Appl Genet. 2002;104:860-7.

38. Kubis SE, Castilho AMM, Vershinin AV, Heslop-Harrison JS. Retroelements, transposons and methylation status in the genome of oil palm (Elaeis guineensis) and the relationship to somaclonal variation. Plant Mol Biol. 2003:52:69-79.

39. Singh R, Ong-Abdullah M, Low E-TL, Manaf MAA, Rosli R, Nookiah R, et al. Oil palm genome sequence reveals divergence of interfertile species in old and new worlds. Nature. 2013;500:335-9.

40. Lerat E. Identifying repeats and transposable elements in sequenced genomes: how to find your way through the dense forest of programs. Heredity. 2010;104:520-33.

41. Bergman CM, Quesneville H. Discovering and detecting transposable elements in genome sequences. Brief Bioinform. 2007:8:382-92.

42. Flutre $\mathrm{T}$, Duprat $\mathrm{E}$, Feuillet $\mathrm{C}$, Quesneville $\mathrm{H}$. Considering transposable element diversification in de novo annotation approaches. PLoS One. 2011;6:e16526.

43. Jurka J, Kapitonov W, Pavlicek A, Klonowski P, Kohany O, Walichiewicz J. Repbase update, a database of eukaryotic repetitive elements. Cytogenet Genome Res. 2005;110:462-7.

44. Lloréns C, Futami R, Bezemer D, Moya A. The Gypsy Database (GyDB) of mobile genetic elements. Nucleic Acids Res. 2008;36 suppl 1:D38-46.

45. Smit A, Hubley R, Green P. RepeatMasker 4.0. Seattle, WA: Institute for Systems Biology; 2013.

46. McCarthy EM, MCDonald JF. LTR_STRUC: a novel search and identification program for LTR retrotransposons. Bioinformatics. 2003;19:362-7.

47. Chaparro C, Gayraud T, de Souza RF, Domingues DS, Akaffou S, Vanzela ALL, et al. Terminal-repeat retrotransposons with GAG domain in plant genomes: a new testimony on the complex world of transposable elements. Genome Biol Evol. 2015;7:493-504.

48. SanMiguel P, Tikhonov A, Jin Y-K, Motchoulskaia N, Zakharov D, MelakeBerhan $A$, et al. Nested retrotransposons in the intergenic regions of the maize genome. Science. 1996;274:765-8.

49. Charles M, Belcram H, Just J, Huneau C, Viollet A, Couloux A, et al. Dynamics and differential proliferation of transposable elements during the evolution of the B and A genomes of wheat. Genetics. 2008;180:1071-86.

50. Vitte C, Ishii T, Lamy F, Brar D, Panaud O. Genomic paleontology provides evidence for two distinct origins of Asian rice (Oryza sativa L.). Mol Genet Genomics. 2004;272:504-11.

51. Noma K, Nakajima R, Ohtsubo H, Ohtsubo E. RIRE1, a retrotransposon from wild rice Oryza australiensis. Genes Genet Syst. 1997;72:131-40.

52. Meerow AW, Noblick L, Salas-Leiva DE, Sanchez V, Francisco-Ortega J, Jestrow B, et al. Phylogeny and historical biogeography of the cocosoid palms (Arecaceae, Arecoideae, Cocoseae) inferred from sequences of six WRKY gene family loci. Cladistics. 2014;31: 1-26.

53. Baker WJ, Couvreur TLP. Global biogeography and diversification of palms sheds light on the evolution of tropical lineages. II. Diversification history and origin of regional assemblages. J Biogeogr. 2013;40:286-98.

54. Devos KM, Brown JKM, Bennetzen JL. Genome size reduction through illegitimate recombination counteracts genome expansion in arabidopsis. Genome Res. 2002;12:1075-9.

55. Ma J, Devos KM, Bennetzen JL. Analyses of LTR-retrotransposon structures reveal recent and rapid genomic dna loss in rice. Genome Res. 2004;14:860-9.

56. Vitte C, Panaud O. LTR retrotransposons and flowering plant genome size: emergence of the increase/decrease model. Cytogenet Genome Res. 2005;110:91-107.

57. Ma J, Bennetzen JL. Rapid recent growth and divergence of rice nuclear genomes. Proc Natl Acad Sci U S A. 2004;101:12404-10.

58. Brunner S, Fengler K, Morgante M, Tingey S, Rafalski A. Evolution of DNA sequence nonhomologies among maize inbreds. Plant Cell. 2005;17:343-60.
59. Denoeud F, Carretero-Paulet L, Dereeper A, Droc G, Guyot R, Pietrella M, et al. The coffee genome provides insight into the convergent evolution of caffeine biosynthesis. Science. 2014;345:1181-4.

60. D'Hont A, Denoeud F, Aury J-M, Baurens F-C, Carreel F, Garsmeur O, et al. The banana (Musa acuminata) genome and the evolution of monocotyledonous plants. Nature. 2012;488:213-7.

61. Barghini E, Natali L, Giordani T, Cossu RM, Scalabrin S, Cattonaro F, et al. LTR retrotransposon dynamics in the evolution of the olive (Olea europaea) genome. DNA Res. 2015;22:91-100.

62. Kalendar R, Vicient CM, Peleg O, Anamthawat-Jonsson K, Bolshoy A, Schulman AH. Large retrotransposon derivatives: abundant, conserved but nonautonomous retroelements of barley and related genomes. Genetics. 2004; 166:1437-50

63. Witte C-P, Le QH, Bureau T, Kumar A. Terminal-repeat retrotransposons in miniature (TRIM) are involved in restructuring plant genomes. Proc Natl Acad Sci. 2001;98:13778-83.

64. Martínez G, Slotkin RK. Developmental relaxation of transposable element silencing in plants: functional or byproduct? Curr Opin Plant Biol. 2012;15:496-502.

65. Ohtsu K, Smith MB, Emrich SJ, Borsuk LA, Zhou R, Chen T, et al. Global gene expression analysis of the shoot apical meristem of maize (Zea mays L.). Plant J. 2007:52:391-404

66. Vicient CM. Transcriptional activity of transposable elements in maize. BMC Genomics. 2010;11:601.

67. Tamaki S, Tsuji H, Matsumoto A, Fujita A, Shimatani Z, Terada R, et al. FT-like proteins induce transposon silencing in the shoot apex during floral induction in rice. Proc Natl Acad Sci. 2015;112:E901-10.

68. Tsuchiya T, Eulgem T. An alternative polyadenylation mechanism coopted to the Arabidopsis RPP7 gene through intronic retrotransposon domestication. Proc Natl Acad Sci. 2013;110:E3535-43.

69. Le TN, Miyazaki Y, Takuno S, Saze H. Epigenetic regulation of intragenic transposable elements impacts gene transcription in Arabidopsis thaliana. Nucleic Acids Res. 2015:43:3911-21.

70. Jaligot E, Hooi WY, Debladis E, Richaud F, Beulé T, Collin M, et al. DNA methylation and expression of the EgDEF1 gene and neighboring retrotransposons in mantled somaclonal variants of oil palm. PLoS One. 2014;9:e91896.

71. Altschul SF, Madden TL, Schäffer AA, Zhang J, Zhang Z, Miller W, et al. Gapped BLAST and PSI-BLAST: a new generation of protein database search programs. Nucleic Acids Res. 1997;25:3389-402.

72. Birney E, Clamp M, Durbin R. GeneWise and Genomewise. Genome Res. 2004;14:988-95

73. Li W, Godzik A. Cd-hit: a fast program for clustering and comparing large sets of protein or nucleotide sequences. Bioinformatics. 2006;22:1658-9.

74. Kohany O, Gentles AJ, Hankus L, Jurka J. Annotation, submission and screening of repetitive elements in Repbase: RepbaseSubmitter and Censor. BMC Bioinf. 2006;7:474.

75. Krzywinski M, Schein J, Birol I, Connors J, Gascoyne R, Horsman D, et al. Circos: an information aesthetic for comparative genomics. Genome Res. 2009;19:1639-45.

76. Andrews S. FastQC: A quality control tool for high throughput sequence data. 2010. http://www.bioinformatics.babraham.ac.uk/projects/fastqc/. Accessed Oct 12, 2015.

77. Martin M. Cutadapt removes adapter sequences from high-throughput sequencing reads. EMBnet J. 2011;17:10-2.

78. Li H. Aligning sequence reads, clone sequences and assembly contigs with BWA-MEM. ArXiv Prepr ArXiv13033997. 2013.

79. Li H, Handsaker B, Wysoker A, Fennell T, Ruan J, Homer N, et al. The sequence alignment/map format and SAMtools. Bioinformatics. 2009;25:2078-9.

80. Robinson MD, McCarthy DJ, Smyth GK. edgeR: a Bioconductor package for differential expression analysis of digital gene expression data. Bioinformatics. 2010;26:139-40.

81. Kolde R. pheatmap: Pretty heatmaps. R package version 0.6.1. 2013. http://CRAN.R-project.org/packageepheatmap. Accessed Oct 12, 2015 